\title{
Prediction and Imitation of Other's Motions by Reusing Own Forward-Inverse Model in Robots
}

\author{
Tetsuya Ogata, Ryunosuke Yokoya, Jun Tani, Kazunori Komatani, and Hiroshi G. Okuno
}

\begin{abstract}
This paper proposes a model that enables a robot to predict and imitate the motions of another by reusing its body forward-inverse model. Our model includes three approaches: (i) projection of a self-forward model for predicting phenomena in the external environment (other individuals), (ii) "triadic relation" that is mediation by a physical object between self and others, (iii) introduction of infant imitation by a parent. The Recurrent Neural Network with Parametric Bias (RNNPB) model is used as the robot's self forward-inverse model. A group of hierarchical neural networks are attached to the RNNPB model as "conversion modules". Experiments demonstrated that a robot with our model could imitate a human's motions by translating the viewpoint. It could also discriminate known/unknown motions appropriately, and associate whole motion dynamics from only one motion snap image.
\end{abstract}

\section{INTRODUCTION}

Like living things, agents fundamentally have to acquire a model of their external environment. Behavioral decisions need the prediction of environmental changes caused by various dynamic objects such as caregivers and/or predators. The learning load for modeling these different objects is huge, but it could be drastically reduced if these objects have common dynamic features and the agent could extract them effectively.

Here, we focus on a mechanism using the self-internal model of the agent for extracting such features and reusing them. In other words, dynamic objects in the external environment are replicated by transforming and reusing a forward-inverse model of the agent. Here "translation" includes various senses such as the viewpoint, body configuration, and situation (context). The agent is expected to be able to acquire models of dynamic objects with a low learning cost.

We focus especially on viewpoint translation, and propose a hypothetical model of parent-infant imitation as an example. Demiris et al. [1] and Makino et al. [2] discussed the forward model predicting another's behavior by reusing the self-internal model. However, there have been little discussion of reusing the self-model, including viewpoint translation.

Our final goal is to model the developmental process of imitation from the standpoint of cognitive developmental robotics [3]. In this paper, we propose a constructive model

T. Ogata, R. Yokoya, K. Komatani, and H. G. Okuno is with the Department of Intelligence Science and Technology, Graduate School of Informatics, Kyoto University, Kyoto, Japan \{ogata, komatani, okuno\}@kuis.kyoto-u.ac.jp

J. Tani is with the Brain Science Institute, RIKEN, Saitama, Japan tani@brain.riken.jp in which a robot imitates an observed motion by predicting it using a self-model. In other words, the robot infant imitates the other individual by creating a prediction model for the other's motions. We have already proposed a method for creating another-model to predict other individuals' motions, which consisted of three phases [7]. In [7], four neural networks were given for translating the robot's view-point. In this paper, the previous method is expanded by adding a fourth phase where the robot infant imitates the other individual using multiple neural networks trained by mixture-of-experts manner. Our model implemented in the robot consists of two kinds of neural network models.

Section II describes two hypothetical conditions applied to our model from the findings of cognitive science. Then, the details of the imitation model reflecting the self-model onto external objects are described. Section III explains the implementation of the robot hardware and the neural network model. Section IV presents the imitation experiments and the obtained results. Furthermore, two characteristics of our model are described: the ability to discriminate known and unknown motions and the association of a whole motion from a static image. Section $\mathrm{V}$ concludes the paper and discusses the future works.

\section{CReating AN Other Model By Projecting SELF-MODEL}

\section{A. Proposed Method}

We introduce two hypothetical conditions into our model, focusing on the developmental process of human infants.

1) Triadic Relation

As reported by Jones, infants begin to imitate the actions of visible parts, e.g., hands or feet, before they can imitate other concealed parts, e.g., tongues [4]. Trevarthen et al. insists that interaction in a "triadic relation", involving an infant, objects, and parents, is essential for imitation development [5]. We introduce an object handled by the robot infant and human parents to replicate the "triadic relation". The task of our robot is to imitate the object's motions shown by a human caregiver.

2) Introduction of "parent's imitation of infant motions"

Piaget noted in his seminal work that a parent's reaction to an infant, including imitating the infant's motions, is critical for the cognitive development of infants [6]. Jones explains the developmental process of imitation based on "parent's imitation of infant motions" in the frame of "associative learning" [4]. Actually, it is plausible that almost all a parent's reactions to an infant's motions are imitations.

Here, we give an overview of our imitation method. 
(i) Recurrent Neural Network (RNN) model

We use a kind of recurrent neural network called the RNNPB (RNN with parametric bias) [8] shown in the middle of Fig. 1 as the forward-inverse model of the robot infant. The RNNPB enables the robot infant to acquire the relations between the robot's motions and the resulting object's motions. We used the RNNPB model here. No matter what forward-inverse model is used, the generality of our model is not lost.

(ii) Hierarchical neural networks

The other-model is organized by attaching multiple hierarchical neural networks (HNNs) as described in Fig. 1. These HNNs called "conversion modules" are used for bi-directional conversion between the subjective and objective information indicated as $I_{s}$ and $I_{o}$, respectively. In our experimental setup, object motion is a conversion target. The conversion modules could be replaced by a neural network with parametric bias (PB) like RNNPB mentioned above. Here, we used multiple neural networks trained in the mixture-of-experts manner [9]. No matter what translation module, e.g. neural network with parametric bias, is used, the generality of our model is not lost.

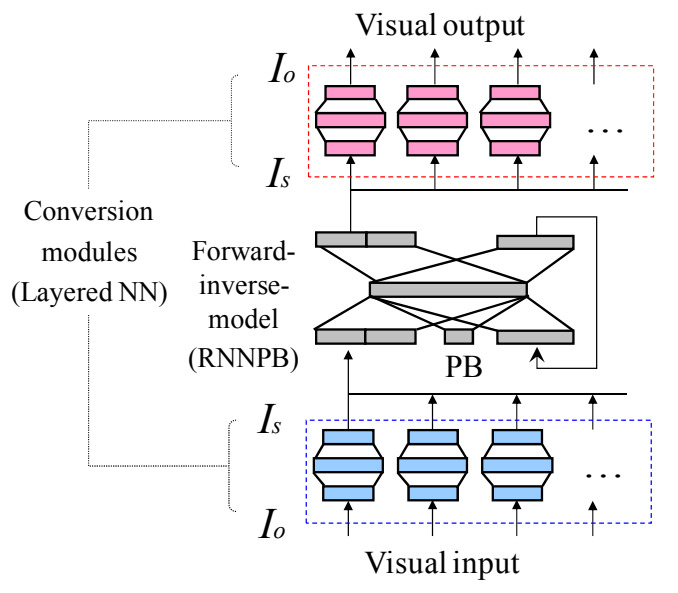

Figure 1 Proposed Model for Translating Viewpoint.

Our developmental model of imitation consists of four phases (see Fig. 2). This process progresses seamlessly on the outside; that is, the robot infant and human caregiver imitate mutually. Note that the robot focuses on the object's motion all the time. This is summarized as follows.

Phase 1: Acquiring Self-model (Infant Body Babbling)

The robot accumulates data about its arm motions and object motions while it is manipulating an object using multiple motions. The self-model, the RNNPB model, is then trained with the data. The relations between the robot's motions and object's motions are acquired as dynamical attractors in the RNN in this phase.

Phase 2: Training of Conversion Modules (Parent's imitation of infant motions):

Soon after the robot has first manipulated the object, the human caregiver imitates it at multiple positions $(0,90,180$, and $270 \mathrm{deg}$ ) as a representative reaction of parents to infant motions. While the human is manipulating the object, the robot accumulates the observed data. The conversion modules are trained with the data so that the other-model with the RNNPB and conversion modules can predict the object's motion. The training is carried out by utilizing a mixture-of-experts algorithm. In the second and third phases, we assume that the robot knows it is being imitated by the human.

Phase 3: Identification of Conversion Module (Parent's imitation of infant motions)

In the same way as in the previous phase, the robot accumulates data while it is observing the human's imitative motions at a certain position. The robot predicts the observed motions by using the other-model and selects the conversion module that can predict the motions most accurately.

Phase 4: Generation of Imitative Motion (Infant's imitation of parent motions):

The robot accumulates data while it is observing the human's motion at the same position as in the third phase. The robot then recognizes the motion being presented through the conversion module selected in the previous phase, and it generates an imitative motion using the RNNPB.

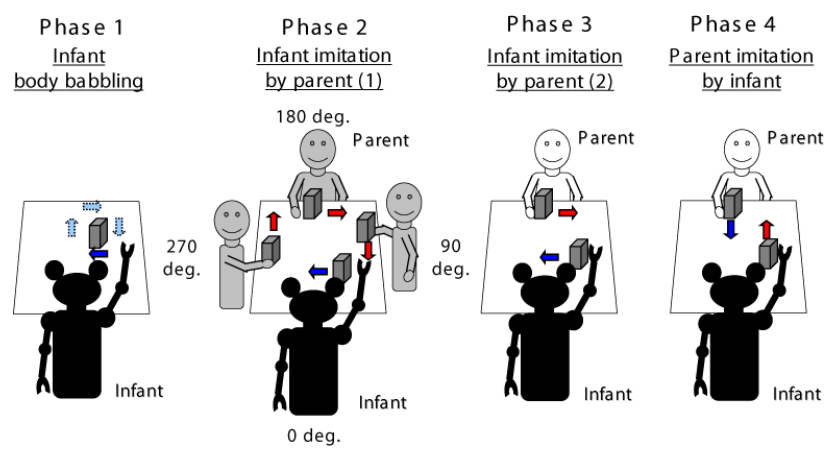

Figure 2 Proposed Process of Imitation Development.

\section{B. Self-model and Other-model}

This section describes the learning models used in our method and their learning algorithms.

1) RNNPB and Its Learning Algorithm

This section describes the architecture and learning algorithm for the RNNPB model. This model is a forwarding forward model proposed by Tani and Ito [8]. It works as a prediction system: its input is the current sensory state $S(t)$ and its output is the predicted sensory state $S(t+1)$ in the next step. This model has the same architecture as the Jodan type recurrent neural network model except for the PB nodes in the input layer. Unlike the other input nodes, these PB nodes take a constant value throughout each time sequence. After learning, the RNNPB model self-organizes the PB values. Here, the PB value becomes a kind of hyper parameters of the RNN that generate dynamics of fixed-point and/or as cyclic attractors.

$$
\begin{aligned}
& \delta \rho_{i}=\varepsilon \sum_{t=0}^{T} \delta_{t}^{b p^{i}} \\
& p_{t}=\operatorname{sigmoid}\left(\rho_{t} / \zeta\right)
\end{aligned}
$$


where $\varepsilon$ is a constant, $\rho_{t}$ is the internal value of the $\mathrm{PB}$ node at $t, p_{t}$ is the PB value of the PB node at $t, \delta^{b p}{ }_{t}$ is the delta error back-propagated from the output nodes to the PB nodes, $T$ is the sensory sequence length. In Eq. (1), the delta errors are integrated errors in all the steps. In Eq. (2), the current PB values are obtained from the sigmoidal outputs of the internal values. Based on these equations, a unique PB value is calculated for each time sequence. In the first phase, the weights and PB values of the RNNPB model are calculated simultaneously. The input data for the RNNPB model were motor and visual information in this work.

2) Learning of Conversion Modules by Mixture of Experts

In our previous work [7], the correspondence relations between the conversion modules and the human positions were prepared in advance. On the other hand, in the study reported here, a learning algorithm called the "mixture of experts" was used for the self-organization of the conversion modules in the second phase. The learning algorithm is as follows.

a) Multiple conversion modules $M_{i}\left(i=0,1, \ldots, N_{M}\right)$ are prepared, and the weights of all modules are randomly initialized.

b) Learning datum $D_{L}$ is randomly selected from data set $D_{j}(j$ $\left.=0,1, \ldots, N_{D}\right)$.

c) All the conversion modules predict $D_{L}$, and conversion module $M_{\min }$ with the lowest prediction error is selected.

d) The weights of $M_{\min }$ are trained using $D_{L}$. Only the weights of the module are updated by back propagation through time (BPTT) without updating the weights of the RNNPB.

Steps b), c), and d) are repeated until the prediction errors for all data have converged.

Here, the PB value of the RNNPB is the same as the one in the first phase. The PB value represents the motion, and we assume that the robot knows what kind of manipulation the parent did, that is, the parent's imitation of the infant's motion.

The initial arm motor values are then input to the motor input layer at time 0 , and the outputs are obtained in the closed looping mode from time 1; the outputs in the motor output layer at time $t-1$ are input to the motor input layer at time $t$. To put this simply, the motor input layer plays the same role as the context layer does. Moreover, the conversion module is identified using the mixture-of-experts algorithm mentioned above.

\section{3) Method of Generating Imitative Motion}

In the fourth phase, the robot recognizes the observed motion and generates imitative motion from its own perspective by translating the viewpoint. The RNNPB with the conversion module selected in the third phase calculates the PB values by BPTT without updating the weights. An imitative motion is generated by inputting the acquired $\mathrm{PB}$ values into the RNNPB.

\section{IMPLEMENTATION OF IMITATION MODEL INTO HUMAN-ROBOT INTERACTIONS}

\section{A. Humanoid Robot Robovie-IIs and Target Object}

Our experimental platform was a humanoid robot, Robovie-IIs (Fig. 3), a refined version of Robovie-II developed at ATR [10]. Robovie has three degrees of freedom (DOFs) in its neck and four DOFs in each arm. Each motor angle value is measured with potentiometers. It also has stereoscopic CCD cameras installed in its head. The manipulation target was a box-shaped object. The top of the object was separated into two colors, red and blue, shown in Fig. 4.

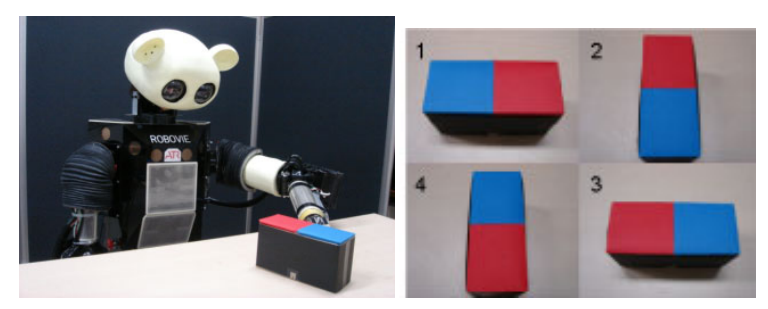

Figure 3 Robovie-IIs. Figure 4 Initial Postures of Target Object.

\section{B. Experimental System}

After the camera and the motors have collected image and joint data, the RNNPB self-model and the conversion modules are trained with the data off-line. The RNNPB model consists of 54 neurons: 7 in the input layer, 35 in the middle layer, 10 in the context layer, and 2 as parametric bias. Although the number of conversion modules for modeling was previously arbitrary, in this work, eight sets of conversion modules were used. Each conversion module consisted of 8 neurons: 4 in the input layer and 4 in the middle layer. The input for the modules was visual data only. The following sensory data were accumulated from the experiment to use in the model.

\section{a) Visual Information (four units)}

The trajectory of an object was accumulated from a CCD camera with a resolution of $500 \times 400$ pixels. The center position of each colored top face, i.e., the X-Y coordinates in the camera ([0-1]), was estimated by extracting the object from the color information; the visual information had four dimensions. The initial position of the object was the center of the camera view in all phases. Only the left-eye camera was used.

\section{b) Motor Information (three units)}

The left arm (3 DOFs: roll and yaw axes of the shoulder and pitch axis of the elbow) was used. Note that the pitch axis of the shoulder and the neck motors were fixed in the experiment. The initial arm poses were the same in all motions. These values were synchronized between different modalities, and were normalized in the range [0.1 - 0.9] based on the maximum and minimum values. The sensory data were stored every $800 \mathrm{~ms}$. for each manipulation, and they were ten steps in time length; that is, the duration of each trial was $8 \mathrm{~s}$. 


\section{IMITATION EXPERIMENT}

Two kinds of experiments were conducted to verify our hypothetical model.

\section{A. Imitation of Known Motions \\ 1) Target Task}

There were four kinds of manipulation: parallel translation from the left to the right (Motion 1: LtoR), rotation to the left by pushing the right side of the object (Motion 2: Lrot), parallel translation from the front to the back (Motion 3: FtoB), and rotation to the right by pushing the left side of the object (Motion 4: Rrot). Only motion 4 was not used for learning the conversion modules in the second phase.

2) Procedure

Phase 1) Learning of Self-model

The robot first performed the four motions described in section IV-A.1). All motions began from all of the four object postures (see Fig. 4) to prevent the robot from selecting a conversion module using only the initial posture of the object. A total of sixteen sets of data were collected in the first phase. The RNNPB model was then simultaneously trained with the collected data. In the second and third phases, both the robot and the human began the motions from the initial posture of object 1 (see the upper left of Fig. 4).

Phase 2) Training of Conversion Modules

The robot observed three types of manipulations, i.e., motions 1,2 , and 3 , presented by one human subject. The human showed the motions once at four positions, 0 deg., 90 deg., 180 deg., and 270 deg to the robot (see Fig. 2). These angles represent the parent's position in the counterclockwise direction relative to the initial position of the object. The robot collected 12 sets of visual data. Eight sets of conversion modules $M_{i}(i=0,1, \ldots, 7)$ were trained with the collected sensory data. It takes about two hours using a normal PC. Note again that the PB values corresponding to the observed manipulations acquired in the first phase were input to the other-model in the second and third phases.

Phase 3: Identification of Conversion Module

The robot observed motions 1, 2, 3, and 4 presented by the human parent at four positions: it accumulated 16 sets of visual data. The robot selected the conversion module that could predict the motions most accurately for each datum. It takes under one second.

Phase 4: Generation of Imitative Motion

The robot observed motions 1, 2,3, and 4 presented by the human parent at four positions: it accumulated sixteen sets of visual data. The other-model calculated PB values for each datum by using the conversion modules selected in the third phase. The robot finally generated imitative motions by inputting acquired $\mathrm{PB}$ values and the initial motor values to the RNNPB.

Table 1 Selected Conversion Modules.

\begin{tabular}{|c|c|c|c|c|}
\hline Manipulation/position & $0 \mathrm{deg}$ & $90 \mathrm{deg}$ & $180 \mathrm{deg}$ & $270 \mathrm{deg}$ \\
\hline LtoR & $M_{1}$ & $M_{2}$ & $M_{5}$ & $M_{7}$ \\
\hline Lrot & $M_{1}$ & $M_{2}$ & $M_{0}$ & $M_{4}$ \\
\hline FtoB & $M_{1}$ & $M_{2}$ & $M_{5}$ & $M_{4}$ \\
\hline Rrot & $M_{1}$ & $M_{2}$ & $M_{5}$ & $M_{4}$ \\
\hline
\end{tabular}

3) Results

a) Acquiring View-Transformation in Conversion Modules

The conversion modules selected for all manipulations in the third phase are listed in Table 1. Modules $M_{1}, M_{2}, M_{5}$, and $M_{4}$ acquired viewing transformations corresponding to $0 \mathrm{deg}$., 90 deg., 180 deg., and 270 deg. We confirmed that the conversion modules were selected appropriately, even in the case of the motion 4, Rrot, which was not used for training conversion modules. Note that our model can predict motions even if the human stands at any positions (directions) by linearly combining the output of the translation modules.

b) Imitative Motions by Robot

In the fourth phase, the robot could generate appropriate imitative motions from the human's perspective for all the observed motions. Photographs of typical imitation experiment are shown in Fig. 5. The results demonstrate that the robot infant could imitate all human motions from its own perspective by reusing its self-model.
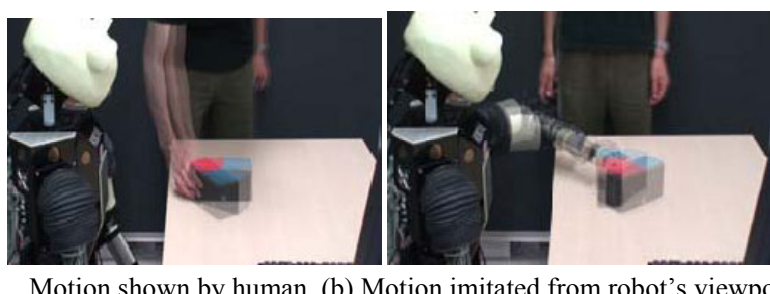

(a) Motion shown by human (b) Motion imitated from robot's viewpoint Figure 5 Photographs of Imitation Experiment.

\section{c) Analysis of PB Space}

The two dimensional PB space acquired in the first and fourth phases is shown in Fig. 6. Here, the PB values for the "original (phase 1)" correspond to manipulation of which the initial posture is 'object 1' in Fig. 4. Although, there is little data spread in the PB values of motion 4, Rrot, which was not used for training the conversion modules, the $\mathrm{PB}$ values of same motions form clusters in the space. This indicates that the shown motions were recognized as being the same as the robot's motions even though they were from different viewpoints.

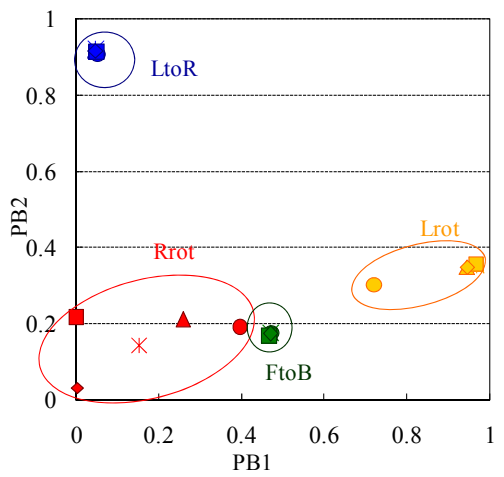

\footnotetext{
$<$ Style $>$

*: Original (Phase 1), Circle: 0 deg (Phase 4), Triangle: $90 \mathrm{deg}$

(Phase 4), Box: 180 deg (Phase 4), Diamond: 270 deg (Phase 4)

$<$ Color $>$

Blue: LtoR, Yellow: Lrot, Green: FtoB, Red: Rrot
}

Figure 6 Acquired PB Space. 
B. Judgment of Known and Unknown Motions by Repeating RNN Association

Section IV-A showed that the robot can imitate the known motions experienced in the first phase by reusing its self-model. However, when the human parent shows a novel manipulation that was not included in the first phase, the robot cannot recognize/imitate it. We think that "the others" are "the parts of the world that can be predicted and imitated by self." In this sense, the judgment ability of known (predictable) and unknown (unpredictable) motions has strong relations to the ability of "discovering others".

Since neural network models have strong generalization ability, it is usually difficult to judge whether it knows the input data. Here, we propose a method that repeats the RNNPB's association, which is $\mathrm{PB}$ recognition and sequence generation by closed loops.

1) Task

A novel motion was added as an unknown motion: rotation to the left by pulling the left side of the object (Motion 5: Lrot2). Note that the object trajectories of Lrot and Lrot 2 were quite different. Motion 5 was not used in the first and second phases, so it was unknown to the robot.

2) Procedure

The first and second phases were conducted in the same way as the experiment in section III-A. In phase 3, the robot observed motions 1-5 presented by the human parent at four positions. It accumulated twenty sets of visual data.

The robot selected the conversion module that could predict the motions most accurately for each datum. Although motion 5 was an unknown motion, a module was tentatively selected by using the prediction error.

In phase 4, the robot infant judged whether the observed motions presented by the human parent are known or unknown.

(a) The other-model tentatively determined a PB value corresponding to each observed motion using the conversion module selected in the third phase.

(b) A virtual motion was associated using the closed-loop of the RNNPB model with the PB value obtained in (a) or (d).

(c) The error $e$ between the observed motion and the virtual motion was calculated.

(d) The other-determined a PB value corresponding to the virtual motion. Then, the procedure returned to $(b)$.

If the observed motion was known, $e$ converged gradually by repeating these processes. Conversely, if the observed motion was unknown, $e$ diverged.

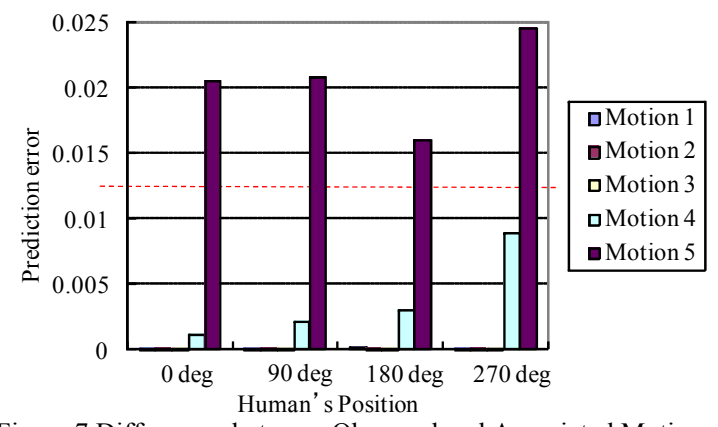

Figure 7 Differences between Observed and Associated Motions.

\section{3) Results}

The prediction errors $e$ obtained by the proposed method are shown in Fig. 7. e means an average squared error at each motion step. The errors for motions 1 and 2 are too small to confirm in the graph. The results of our experiment indicate that our method can discriminate known and unknown motions based on the prediction errors. Decety et al. reported that the routes of processing known and unknown motions were different in the brain [11].

\section{Association of a whole motion from a static image.}

Our model enables the robot infant to associate a whole motion from a static image effectively by reducing the search space into a two-dimensional PB space.

\section{1) Task and Procedure}

The first and second phases were conducted in the same way as the experiment in section III-A. Four static images were randomly selected from snap images of motion 1, 2, 3, and 4, respectively. Moreover, an image of the initial pose was used. Note that the motion association from initial image had ambiguity because the initial pose was common to all motions.

The PB space obtained after training was divided into 100 x 100 lattices. Whole motions were virtually-associated by the RNNPB with the PB values corresponding to these lattices. Then, a motion including an image matching the static image was selected. Note that the search space did not include all the motions that the robot can generate but only some specific motions coded in the PB space: motions experienced in the first phase and their generalized motions.

\section{2) Results}

PB spaces indicating the matching errors between the static images and the initial image by gray scale are shown in Fig. 8. The ' $x$ ' indicates the $P B$ value with the minimum error. By comparing these spaces with Fig. 6, we found that the motion corresponding to each static image was correctly recognized. Furthermore, the motion corresponding to the initial image was not clearly identified because of a small error spreading throughout the whole PB space.

Centroid trajectories of the colors of 'red' and 'blue' in the camera corresponding to the motions generated by the $\mathrm{PB}$ values with minimum error, are shown in Fig. 9. They confirm that the motions that pass through the static images were generated.

\section{CONCLUSION}

This paper proposed a constructive model that enables a robot to imitate other individuals through viewpoint conversion by reusing its own self-model. The other individual is regarded as a dynamic object that can be predicted by projecting/translating a self-model. Two hypothetical conditions were applied to our model of the development process: (i) the "triadic relation" of object-self-other and (ii) a parent's imitation of infant motions. The task was manipulating an object on a table. The RNNPB model was used as the self model (forward-inverse model) for the robot infant, and the HNN was attached to the RNNPB to replicate the oth- 
er-model for the human parent. The experimental results proved that our model is adequate to enable the robot to recognize the manipulations performed by human parents, and to imitate them from its own perspective. Furthermore, two characteristics of our model were described: the ability to discriminate known and unknown motions and the association of a whole motion from a static image.
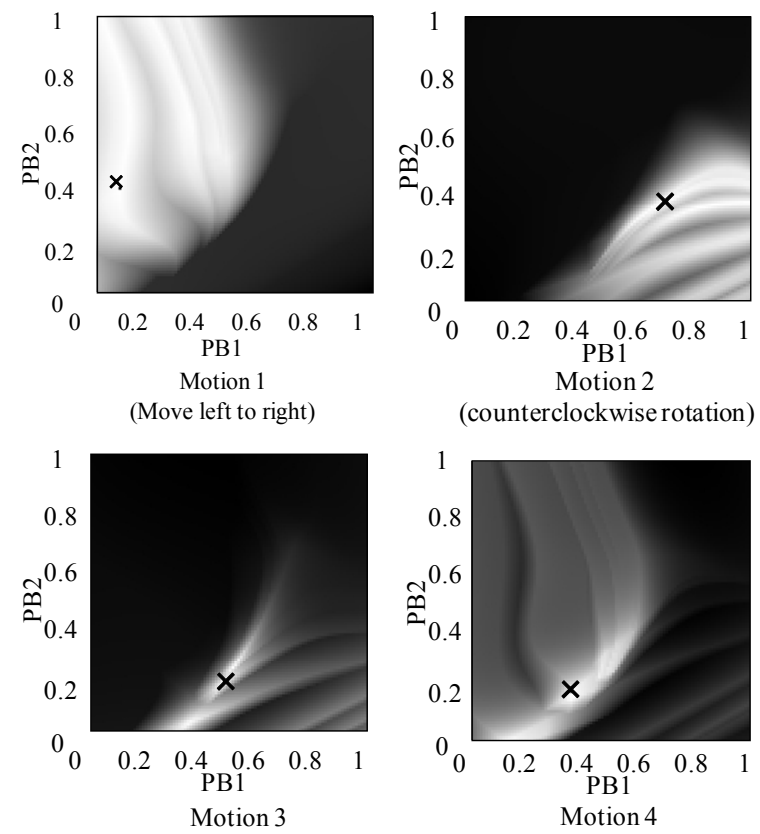

(counterclockwise rotation)

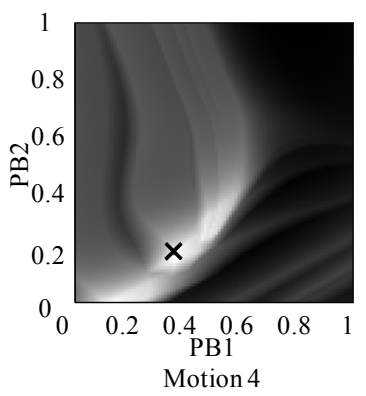

(clockwise rotation)

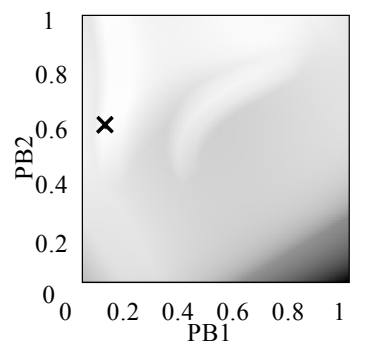

Initial Position

Figure 8 Error Maps between Observed Motions and Motions Associated from Static Images.

As this work is only the first step in modeling the developing processes of imitation, numerous issues still remain to be addressed. The judgment of known/unknown motions could relate to an ability to discover the other individual. This work deals with the ability as the selection of conversion modules. It cannot to be applied to 'mimic' finding of the other individual's body from physical space. This issue is closely related to how to extract the point to be imitated. In this problem, our model relies on the hypothetical condition of 'triadic relation'. The solution to these issues may lead to more skilful imitation. Moreover, we must consider the fact that human communication is built on seamless interaction between the self and others. The development process divided into four phases should be connected smoothly.
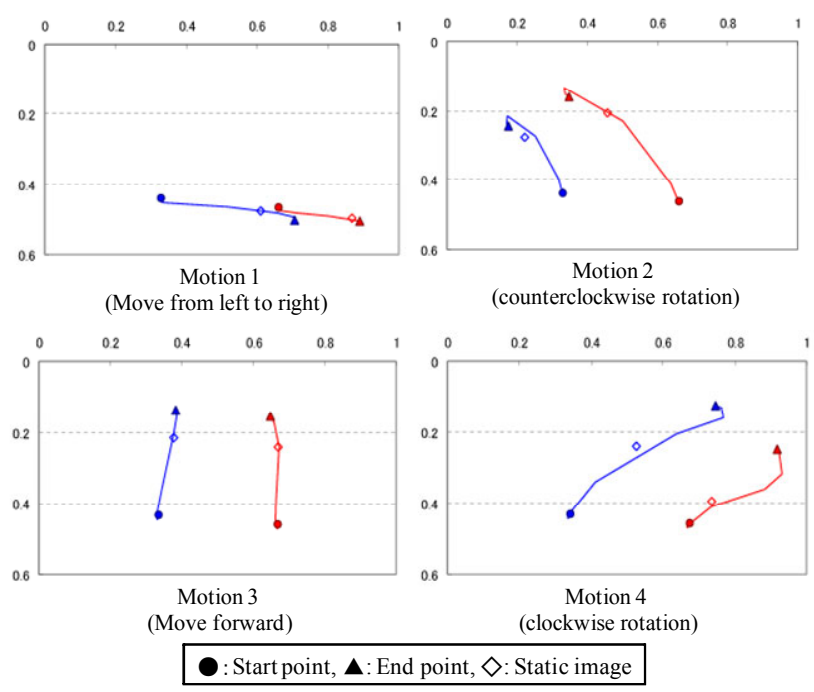

Figure 9 Associated Motion Trajectories from a Static Camera Image

\section{ACKNOWLEDGMENT}

This research was partially supported by the Ministry of Education, Science, Sports and Culture, Grant-in-Aid for Scientific Research (S), and Creative Scientific Research.

\section{REFERENCES}

[1] Y. Demiris and G. Hayes: Imitation as a dual-route process featuring predictive and learning components: a biologically-plausible computational model, Dautenhahn and Nehaniv (Eds.), "Imitation in Animals and Artifacts", MIT Press, chapter 13, pp. 327-361, 2002.

[2] T. Makino and K. Aihara: Self-observation Principle for Estimating the Other's Internal State, Mathematical Engineering Technical Reports METR 2003-36, The University of Tokyo, 2003.

[3] Asada, M., MacDorman, K.F., Ishiguro, H., and Kuniyoshi, Y.: Cognitive developmental robotics as a new paradigm for the design of humanoid robots; Robotics and Autonomous Systems, pp. 185-193 (2001).

[4] S. Jones: Infants Learn to Imitate by Being Imitated; Proc. of IEEE/RSJ Int. Conf. on Development and Learning (2006).

[5] C. Trevarthen and P. Hubley: Secondary intersubjectivity: Confidence, confiding and acts of meaning in the first year, Lock, A.(Eds.), Action, gesture, and symbol: The emergence of language, New York: Academic Press, pp. 183-229, 1978.

[6] J. Piaget: Play, Dreams, and Imitation in Childhood; New York: Norton (1951).

[7] R. Yokoya, T. Ogata, J. Tani, K. Komatani, and H. G. Okuno: Discovery of Other Individuals by Projecting a Self-Model Through Imitation; Proc. IEEE/RSJ IROS2007, pp. 1009-1014 (2007).

[8] J. Tani and M. Ito: Self-Organization of Behavioral Primitives as Multiple Attractor Dynamics: A Robot Experiment; IEEE Trans. on Systems, Man, and Cybernetics Part A: Systems and Humans, Vol. 33, No. 4, pp. 481-488 (2003).

[9] J. Tani and S. Nolfi: Learning to perceive the world as articulated: an approach for hierarchical learning in sensory-motor systems, Neural Networks, Vol. 12, No. 7, pp.1131-1141, 1998.

[10]H. Ishiguro, T. Ono, M. Imai, T. Maeda, T. Kanda, and R. Nakatsu: Robovie: An Interactive Humanoid Robot; International Journal of Industrial Robotics, Vol. 28, No. 6, pp. 498-503 (2001).

[11]J. Decety, J. Grezes, N. Costes, D. Perani, M. Jeannerod, E.Procyk, F. Grassi, and F. Fazio: Brain activity during observation of actions: influence of action content and subject's strategy; Brain, Vol.120, pp.1763-1777 (1997). 